\title{
Performance Analysis for Different Interleavers in Various Modulation Schemes with OFDM over an AWGN Channel
}

\author{
Vineet Chaturvedi ${ }^{1}$, Vivek Kumar Gupta ${ }^{2}$ \\ I(Pursuing M.Tech. Digital Communication, Dehradun Institute of Technology/Uttarakhand Technical University, India) \\ ${ }_{2}^{2}$ (Assistant Professor, E.C.E. Department, Dehradun Institute of Technology/ Uttarakhand Technical University, India)
}

\begin{abstract}
The next generation wireless communications systems need to be of a higher standard in order to provide the customers with the multitude of high quality services they demand. In recent years, Orthogonal Frequency Division Multiplexing (OFDM) has been successfully used in terrestrial digital video broadcasting and showed it is a strong candidate for the modulation technique of future wireless systems. In order to investigate this, a simulation model was created and implemented using MATLAB. This letter focuses on the design of an OFDM system with the interleaver stage for a specific convolutional code operating with OFDM modulation. The interleaver is an important part in the design of a communication system. The performance of different interleavers (Matrix and Random) are compared over AWGN channel with digital and analog modulation schems in OFDM, Numerical results also evidence the importance of the interleaver choice when comparing the performance of different interleavers. Simulation results show that matrix interleaver can be very competitive to random interleaver.
\end{abstract}

Keywords - Additive White Gaussian Noise, Block Interleaver, Convolutional Interleaver, Matrix Interleaver, Orthogonal Frequency Division Multiplexing, Random Interleaver.

\section{INTRODUCTION}

A primary technique which is effective in overcoming error bursts, is interleaving. Interleaving is a standard signal processing technique used in a variety of communication system. The principle of interleaving is that it takes symbols from an input and produces the identical symbols at the output in a different temporal order. The classical use for interleaving is to disperse sequences of bits in a bitstream so as to minimize the effect of burst errors introduced in transmission. Traditionally, the goal of the interleaver in is to break the temporal structure of the channel providing a diversity gain in systems with no CSI at the transmitter, which reduces the bit error rate (BER) at the decoder output [1].

There are two classical kinds of interleavers, commonly referred to as block and convolutional. In a block interleaver, the input data is written along the rows of a memory configured as a matrix, and then read out along the columns. A variation of a block interleaver is a pseudorandom block interleaver, in which data is written in memory in sequential order and read in a pseudorandom order. In a convolutional interleaver, the data is multiplexed into and out of a fixed number of shift registers [2].

In this paper we propose a system model that is motivated by block interleaver, from block interleaver we have taken two most commonly used type of interleavers (i) Matrix interleaver and (ii) Random interleaver.

Using this model, we study the working of block interleaver and identify a certain class of block interleavers to show that which is more optimal with respect to bit error rate for different modulation schemes in orthoonal frequency division multiplexing.

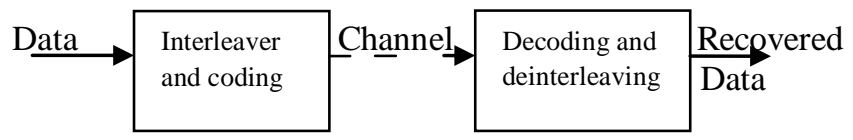

Fig. 1 Interleaving

The Matrix Interleaver performs block interleaving by filling a matrix with the input symbols row by row and then sending the matrix contents to the output port column by column. The Random Interleaver rearranges the elements of its input vector using a random permutation [3].

\section{System model}

Orthogonal frequency division multiplexing (OFDM) is being used widely to achieve high data rates and reduce multipath fading in wireless communications. The transmission of data in this multi-carrier modulation scheme is made by dividing a single wideband stream into several smaller or narrowband parallel bit streams. It is a method to encode digital data on multiple carrier frequencies. It has developed into a popular scheme for wideband digital communication, whether wireless or over copper wires, used in applications such as digital television and audio broadcasting, DSL broadband internet access, wireless networks, and $4 \mathrm{G}[12]$. In a mobile cellular communication system, the users are the mobile transmitters in any particular cell of the system and the receiver resides in the base station of the particular cell [14].

In this section we describe the OFDM system in which the proposed interleavers are used. Convolutional coding is used to encode the information bit stream to be transmitted. After that the interleaving is done to the bits and mapped into constellation symbols with respect of the modulation 
technique and finally modulated into OFDM symbols. An individual carrier is assigned to each narrowband modulated streams. These narrowband channels are orthogonal to each other, and are transmitted simultaneously. Due to this the symbol duration is increased proportionately and reduces the effects of inter-symbol interference (ISI) introduced by multipath Rayleigh-faded environments. The spectra of the subcarriers overlap each other, making OFDM more spectral efficient as opposed to conventional multicarrier communication schemes.

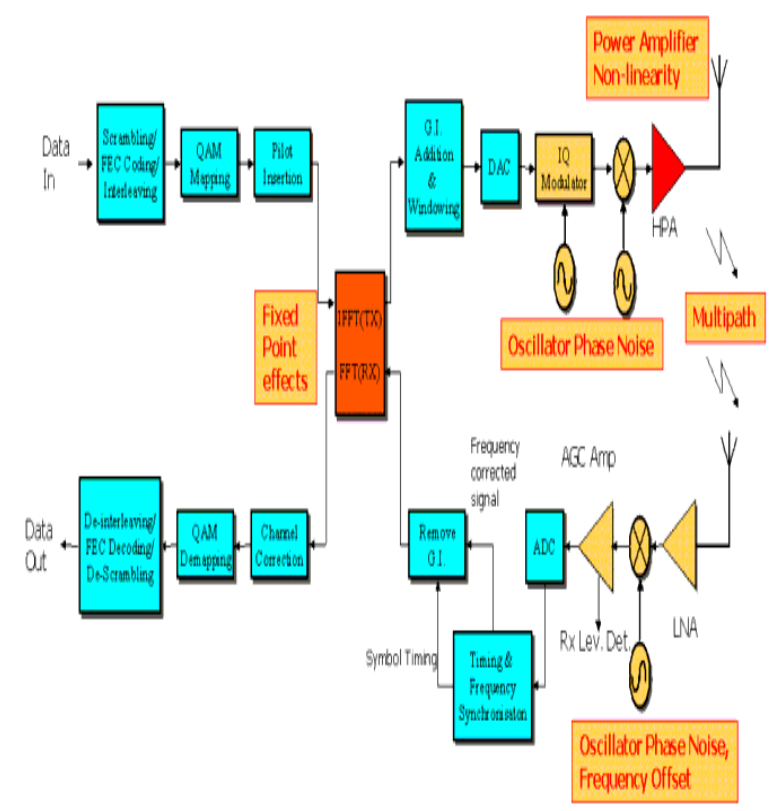

Fig. 2 OFDM block diagram

\section{A. OFDM signal}

The OFDM signal is generated in the complex baseband. To modulate each symbol onto the corresponding subcarrier, various Phase Shift Keying (PSK) or different forms of Quadrature Amplitude Modulation (QAM) are used. Serial to parallel conversion is also done to the data symbols before transmitting it. $\mathrm{N} \pi 2$ is the frequency spacing between adjacent subcarriers, where numbers of subcarriers are defined by N. Inverse Discrete Fourier Transform (IDFT) is used to achieve the frequency spacing easily after implementing the Inverse Fast Fourier Transform (IFFT) operation. Which results, the OFDM symbol generated for an $\mathrm{N}$-subcarrier system translates into $\mathrm{N}$ samples, with the ith sample being

$X i=\sum_{n=0}^{N-1} \operatorname{cn} \exp \left\{j \frac{2 \pi i n}{N}\right\}, 0 \leq i \leq N-1$

At the receiver, Discrete Fourier Transform (DFT) is used to form the frequency domain of corrupted symbols of OFDM signal which were in time domain. Practically fast Fourier transform (FFT) is performed to the received message to recover the original information [4].

\section{B. Interference}

Different replicas of the transmitted symbol reach at the receiver with different times in a multipath environment. The fact behind it is that between transmitter and receiver there are different propagation paths. Due to this time dispersion, in which a particular received symbol is stretched into the one following it. This overlapping of symbols is called inter symbol interference, or ISI. Timing offset is mainly affected by it. Another form of interference is inter-carrier interference or ICI. To maintain orthogonality between the carriers is main factor to demodulate successfully in OFDM.

We demodulate a specific subcarrier $\mathrm{N}$ at its spectral peak, meaning that all the other carriers must have a corresponding zero spectra at the $\mathrm{N}_{\mathrm{th}}$ center frequency (frequency domain perspective). Frequency offsets lead to this criterion not being met. This condition can seriously hinder the performance of our OFDM system. Fig. 3 below shows that when the decision is not taken at the correct center frequency (i.e. peak) of carrier considered, adjacent carriers factor in the decision making, thus reducing the performance of the system [5].

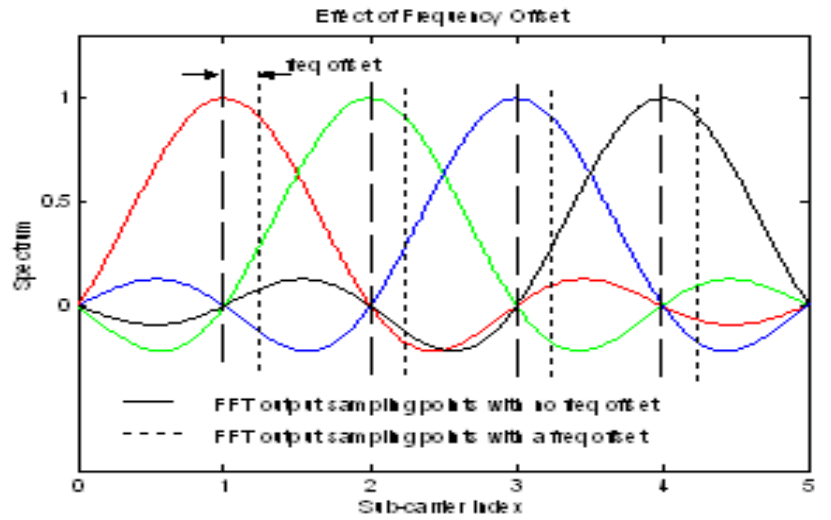

Fig. 3 Effect of frequency offset (maintaining orthogonality)

\section{Cyclic Prefix or Cyclic Extension}

The ISI is a common problem found in high data rate communication. When the transmitted signal interferes with itself then the receiver cannot decode the transmitted correctly. This is because as the data rate increases, the time duration between the consecutive pulses decreases. To avoid ISI, the pulse time duration should be greater than the maximum delay of the channel. Guard interval is provided along with the data period so that the ISI effect observed in the guard interval can be removed afterwards and the data can be retrieved.

One of the most important properties of OFDM transmission is its high level of robustness against multipath delay spread. The level of multipath robustness can be further increased by addition of a guard period between the transmitted symbols. The guard period allows time for multipath signals from the previous symbol to die away before the information from the current symbol is gathered. The most effective use of guard period is cyclic extension of the symbol. If a mirror in time at the end of the symbol waveform is put at the start of the symbol as the guard period, this effectively extends the length of the symbol, while maintaining the orthogonality of the waveform. Using cyclic extended symbol, multipath immunity as well as symbol time synchronization tolerance can be achieved.Transmitting a cyclic prefix of the data during guard interval transforms the linearly convolutive channel. Hence, the channel equalization problem is simplified. Specifically, channel equalization in the frequency domain can be done using one tap filters. This is because the cyclic 
prefixing makes the channel matrix circulant, which is diagonalized by the IDFT and DFT operations.

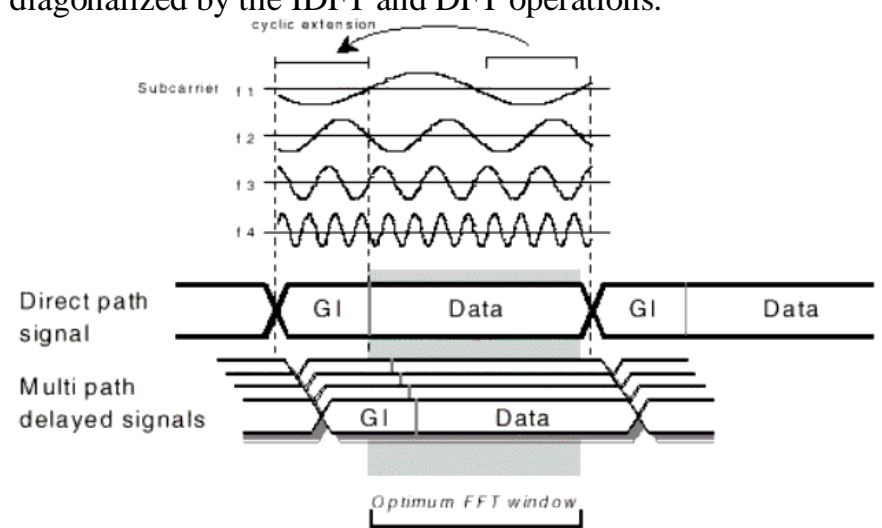

Fig. 4 Added guard interval with symbol

With the cyclic extension, the symbol period is prolonged but it represents slightly extended frequency spectrum. As long as the correct number of samples are to be taken for the decoding it can be taken from anywhere within the extended symbol. Since a complete period is integrated, orthogonality is maintained. Therefore, both ISI and ICI are eliminated.

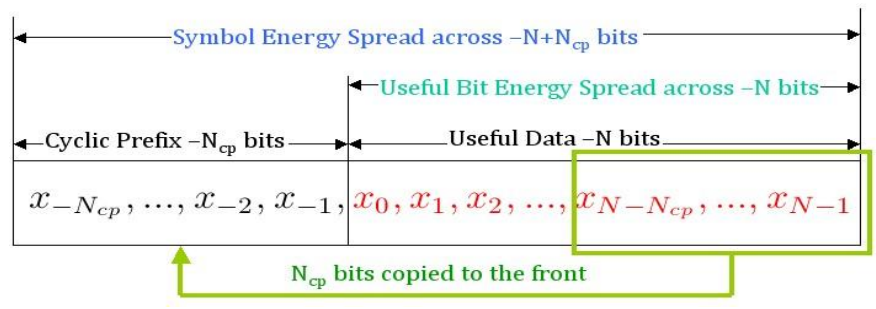

Fig. 5 Cyclic prefix

Although the cyclic prefix introduces a loss in SNR, this may be considered as a small price to pay to mitigate interference. Note that some bandwidth efficiency is lost with the addition of the guard period, but numerous advantages and hence compromising solution is required [5].

\section{Convolution coding}

Convolutional codes, first introduced in 1955 by Elias, it is different from block codes. In a block code, the block of $n$ code digits generated by the encoder in any particular time unit depends only on the block of $k$ input data digits within that time unit. On the other hand, in a convolutional code, the block of $n$ codes digits generated by the encoder in a particular time unit depends not only on the block of $k$ message digits within that time but also on the block data digits with a previous span of $N-1$ time units $(N>1)$. For convolutional codes, $k$ and $n$ are usually small. Convolutional codes can be devised for correcting random errors, burst errors, or both. Encoding is easily implemented by shift registers. As a class, convolutional codes invariably outperform block codes of the same order of complexity [6].

A convolutional code is generated by combining the outputs of a $K$ shift shift registers through the employment of $v$ EXCLUSIVE-OR logic summers. Such a coder is illustrated in Fig. 6 for the case $K=4$ and $v=3$. Here $\mathrm{M}_{1}$ through $\mathrm{M}_{4}$ are 1-bit storage (memory) devices such as flipflops.

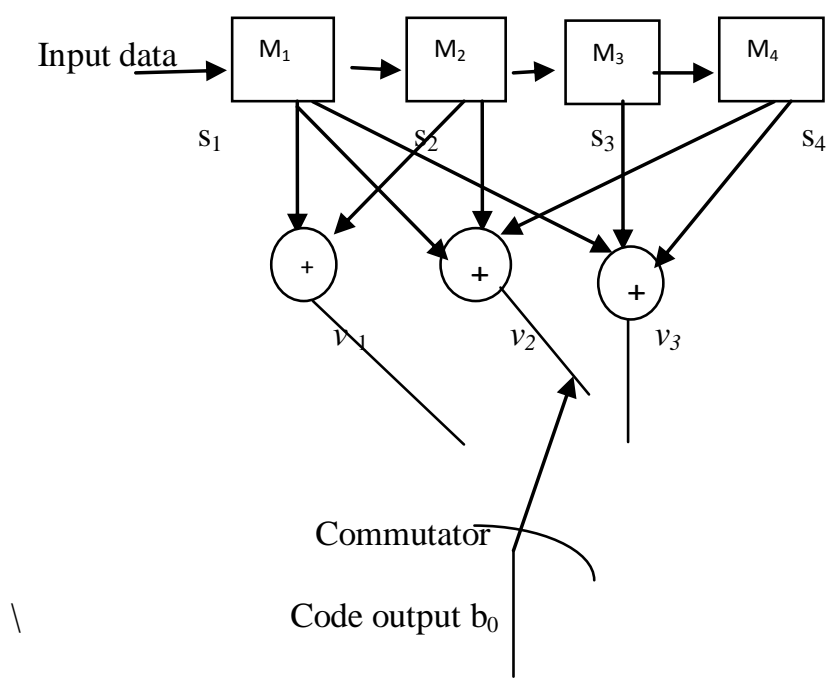

Fig. 6 Block diagram of convolutional encoder

The operation of the encoder proceeds as follows: We assume that initially the shift register is clear. The first bit of the input data is entered into $M_{1}$. During this message bit interval the commutator samples, in turn, the adder outputs $v_{1}, v_{2}$ and $v_{3}$. Thus a single bit yields, in the present case, three coded output bits. The encoder is therefore of rate $1 / 3$. The next message bit then enters $M_{1}$ and the bit initially in $M_{1}$ transfer to $M_{2}$, and the commutator again samples all the $v$ adder outputs. This process continues until eventually the last bit of the message has been entered into $M_{1}$. Thereafter, in order that every message bit may proceed entirely through the shift register, and hence be involved in the complete coding process, enough 0's are added to the message to transfer the last message bit through $M_{4}$, and hence, out of the shift register. The shift register then finds itself in its initial "clear" condition. It may be verified by way of example, that, for the encoder of Fig. 6, if the input bit stream to the encoder is given by the 5 -bit sequence

$$
m=10110
$$

then the coded output bit stream is

$$
c=111010100110001000011000000
$$

If the number of bits in the message stream is $L$, the number of bits in the output code is $v(L+K)$. As a matter of practice however, $L$ is ordinarily very large number while $K$ is a relatively small number. Hence $v(L+K)$ is $=v L$. Thus the number of code bits is $v$ times of number of message bits, $v$ being the number of commutator segments. Accordingly, also, the rate of code is $1 / v$.

The coded output depends on the number $K$ of shift registers stages, on the number of EXCLUSIVE-OR adders used and on the connections of the register stages to the adders. With a view toward searching out optimal encoders, exhaustive computer analysis has been undertaken. Some results, for $1 / 2$ coders (two adders) are given in Table 1 , a 1 in the table indicates a connection to an adder while a 0 indicates that no connection is made. 
TABLE I

Optimum configurations for rate $1 / 2$ convolutional coders Code generator connections $\left(M_{1}, M, \ldots . . M_{k}\right)$

\begin{tabular}{|c|c|c|}
\hline $\begin{array}{c}\text { K(Number of } \\
\text { Register Stages) }\end{array}$ & $v_{1}$ & $v_{2}$ \\
\hline 3 & $1,1,1$ & $1,0,1$ \\
\hline 4 & $1,1,1,1$ & $1,1,0,1$ \\
\hline 5 & $1,1,1,0,1$ & $1,0,0,1,1$ \\
\hline 6 & $1,1,1,0,1,1$ & $1,1,0,0,0,1$ \\
\hline 7 & $1,1,1,1,0,0,1$ & $1,0,1,1,0,1,1$ \\
\hline 8 & $1,1,1,1,1,0,0,1$ & $1,0,1,0,0,1,1,1$ \\
\hline
\end{tabular}

Thus, by way of example, for the case $K=5$, stages $1,2,3$ and 5 should be connected to the adder which generates $v_{1}$ while stages 1,4 and 5 should be connected to the adder which generates $v_{2}[6]$.

\section{Iv. Interleaving}

\section{A. Matrix Interleaving}

Conventionally, interleaving is used to spread out theerrors occurring in bursts like those exhibited in fading channels [1]. In this regard, matrix interleaving, where bits are fed in a matrix row by row and read out column by column, is usually implemented. At the transmitter, the interleaver is used to feed the OFDM symbols with different permutations of the information sequence so that the generated parity sequences can be assumed independent. At the receiver, the deinterleaver is used to randomize the symbols after every decoding step, thus making the iterative decoding more efficient. In this paper, we attempt to provide an in-depth study on the performance of OFDM with matrix interleavers and random interleavers. The column size $n$ is called the depth and the row size $m$ is the span. Such an interleaver is completely defined by $n$ and $m$ and is thus refereed to as $(n, m)$ matrix interleaver. At the deinterleaver, information is written column-wise and read out row-wise. The capability of burst error scattering for the matrix interleaver depends on the values of $n$ and $m$. Since both the interleaver and the deinterleaver are utilized iteratively at the receiver. To test this conjecture, consider an interleaver of size 192 symbols. This size can be arranged in $(192,1),(96,2),(32,6)$, or $(16,12)$ matrices.

\section{B. Random Interleaving}

The simplest type of interleaver merely permutes all lines of the input bit stream randomly. The effect of this random shuffling makes almost all symbols correlated with each other. It rearranges the elements of its input vector using a random permutation. The Random Deinterleaver block rearranges the elements of its input vector using a random permutation. The Initial seed parameter initializes the random number generator that the block uses to determine the permutation. If this block and the Random Interleaver block have the same value for Initial seed, then the two blocks are inverses of each other. The Number of elements parameter indicates how many numbers are in the input vector. If the input is frame-based, then it must be a column vector [3].
For simulation purpose, we based our work on the MATLAB.The program simulates 64 subcarrier OFDM system. Convolutional code generator with rates $1 / 2$ was used. The $1 / 2$ rate code is punctured to get $2 / 3$ code rate. After encoding, interleaving is done to coded bits with (matrix and random interleavers). We used 4 modulation schemes, Binary Phase Shift Keying (BPSK), Quadrature Phase Shift Keying (QPSK), Sixteen Quadrature Amplitude Modulation (16-QAM) and Sixty Four Quadrature Amplitude Modulation (64-QAM). Channel used was additive white Gaussian noise, AWGN. As IFFT size was used 64 so cyclic prefix is $25 \%$ of IFFT size so 16 cyclic prefix were used. A specific average signal to noise ratio is used [10]. The program also uses fine time synchronization, pilot phase tracking, as in IEEE 802.11 standards. At the receiver part the cyclic prefix is removed and pilot symbol is synchronised, demodulation with respect to the modulation scheme is done. After demodulation, deinterleaving and decoding are used to get the original transmitted data. And at last the Bit Error Rate (BER) for both the interleavers is analyzed with the different modulation schemes[11].

\section{A. Simulation Parameters}

\section{TABLE 2}

Simulation Parameters for the programm

\begin{tabular}{|c|c|}
\hline Parameters & Values \\
\hline Data Bits & $\begin{array}{c}\text { Randomly generated( as per the } \\
\text { modulation scheme) }\end{array}$ \\
\hline Modulation Techniques & BPSK,QPSK,(16 and 64QAM) \\
\hline Coding & $\begin{array}{c}\text { Convolutional codes rate } 1 / 2 \text { and } \\
2 / 3\end{array}$ \\
\hline Interleaver & Matrix and Random \\
\hline IFFT & 64 \\
\hline Pilot Symbols & 48 \\
\hline Data Subcarriers & $16(25 \%$ of IFFT size $)$ \\
\hline Cyclic Prefix & $1 / 16$ \\
\hline Guard Interval & $7 \mu$ s \\
\hline Allowed Guard Interval &
\end{tabular}

\section{B. Algorithm of Simulation}

We measured the performance of the interleavers in OFDM through MATLAB simulation. The pocedure to simulate this is given below.

1. Information bits were generated randomly.

2. Information bits were coded using a convolutional encoder with code rate $1 / 2$ with polynomial [01011011, 01111001] and constraint length 7. Puncturing is also done to get the code rate $2 / 3$

3. BPSK, QPSK and different QAM modulation were used to convert the binary bits, 0 and 1 , into complex signals (mapping).

4. Serial to parallel conversion was performed.

5. IFFT was used to generate OFDM signals, zero padding was being done before IFFT.

6. Use parallel to serial convertor to transmit signal serially.

7. Introduce noise to simulate channel errors. We assumed that the signals are transmitted over an AWGN channel. The noise is modeled as a Guassian random variable with zero mean and variance $\sigma^{2}$. The variance of the noise is obtained as

$$
\sigma^{2}=\frac{1}{2 * E b / N O}
$$


which generates a sequence of normally distributed random numbers, where random numbers has zero mean and 1 variance. Thus the received signal at the receiver is :

$$
\mathrm{X}^{\mathrm{e}}=\text { noisy }(\mathrm{X}) \text {. }
$$

Where noisy $(\mathrm{X})$ is the signal corrupted by noise.

8. At the receiver side, reverse operations were performed to decode the received sequence.

9. decoded bits were compared with the original one to count the erroneous bits.

10. BER was calculated and plotted [7].

TABLE 3

Data Rate for different modulation schemes in OFDM[9].

\begin{tabular}{|c|l|l|l|l|l|}
\hline $\begin{array}{l}\text { Data Rate } \\
\text { (Mbps) }\end{array}$ & Modulation & $\begin{array}{l}\text { Coding } \\
\text { Rate }\end{array}$ & $\begin{array}{l}\text { Coded bits } \\
\text { per } \\
\text { subcarrier }\end{array}$ & $\begin{array}{l}\text { Coded } \\
\text { bits per } \\
\text { ofdm } \\
\text { symbol }\end{array}$ & $\begin{array}{l}\text { Informa } \\
\text { tion bits } \\
\text { per } \\
\text { ofdm } \\
\text { symbol }\end{array}$ \\
\hline 6 & BPSK & $1 / 2$ & 1 & 48 & 24 \\
\hline 9 & BPSK & $3 / 4$ & 1 & 48 & 36 \\
\hline 12 & QPSK & $1 / 2$ & 2 & 96 & 48 \\
\hline 18 & QPSK & $3 / 4$ & 2 & 96 & 72 \\
\hline 24 & 16 QAM & $1 / 2$ & 4 & 192 & 96 \\
\hline 36 & 16 QAM & $3 / 4$ & 4 & 192 & 144 \\
\hline 48 & 64 QAM & $2 / 3$ & 6 & 288 & 192 \\
\hline 54 & 64 QAM & $3 / 4$ & 6 & 288 & 216 \\
\hline
\end{tabular}

\section{RESULTS}

A. OFDM with BPSK

The results for matrix and random interleaver with BPSK modulation in OFDM are given below

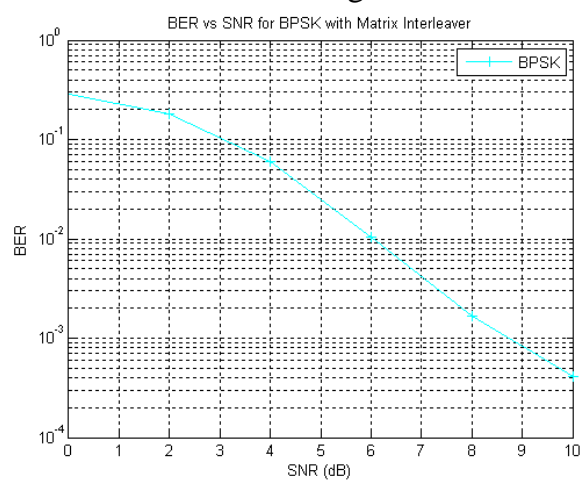

Fig. 7 BER vs. SNR for BPSK with Matrix Interleaving TABLE 4

Numerical result for BPSK in OFDM with Matrix Interleaver

\begin{tabular}{|c|c|}
\hline SNR $(\mathrm{dB})$ & BPSK(Matrix Interleaver) \\
\hline 0 & 0.2896 \\
\hline 2 & 0.1792 \\
\hline 4 & 0.0592 \\
\hline 6 & 0.0104 \\
\hline 8 & 0.0017 \\
\hline 10 & 0 \\
\hline 12 & 0 \\
\hline 14 & 0 \\
\hline 16 & 0 \\
\hline 18 & 0 \\
\hline
\end{tabular}

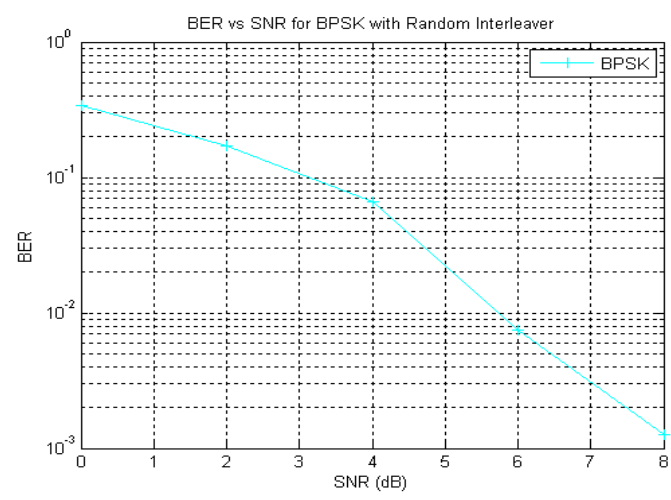

Fig. 8 BER vs. SNR for BPSK with Random Interleaving TABLE 5

Numeriacl results for BPSK with Random Interleaver

\begin{tabular}{|c|c|}
\hline $\mathrm{SNR}(\mathrm{dB})$ & BPSK (Random Interleaving) \\
\hline 0 & 0.3367 \\
\hline 2 & 0.1717 \\
\hline 4 & 0.0667 \\
\hline 6 & 0.0075 \\
\hline 8 & 0.0052 \\
\hline 10 & 0.0013 \\
\hline 12 & 0 \\
\hline 14 & 0 \\
\hline 16 & 0 \\
\hline 18 & 0 \\
\hline
\end{tabular}

\section{B. OFDM with QPSK}

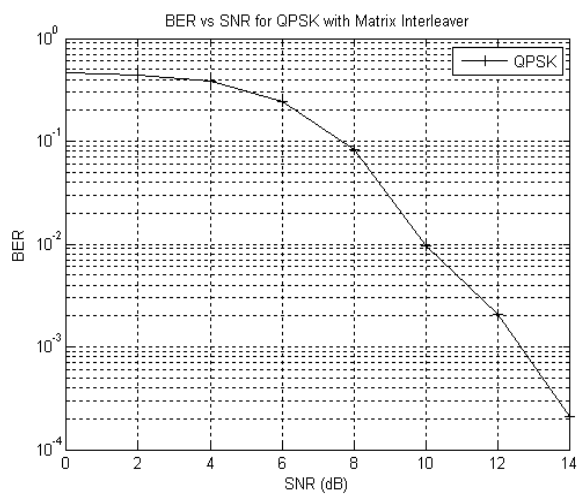

Fig. 9 BER vs. SNR for QPSK in OFDM with Matrix interleaving

TABLE 6

Numeriacl results for QPSK with Matrix Interleaving

\begin{tabular}{|c|c|}
\hline SNR $(\mathrm{dB})$ & QPSK(Matrix interleaver) \\
\hline 0 & 0.4673 \\
\hline 2 & 0.4350 \\
\hline 4 & 0.3835 \\
\hline 6 & 0.2448 \\
\hline 8 & 0.0835 \\
\hline 10 & 0.0096 \\
\hline 12 & 0 \\
\hline 14 & 0 \\
\hline 16 & 0 \\
\hline 18 & 0 \\
\hline
\end{tabular}




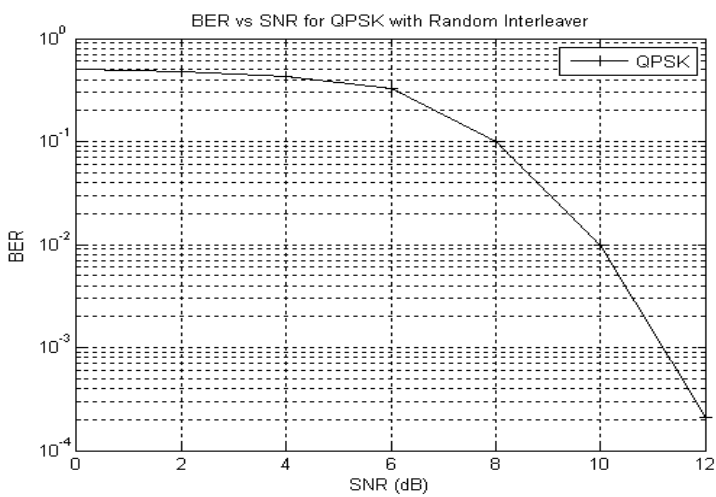

Fig. 10 BER vs. SNR for QPSK in OFDM with Random interleaving

TABLE 7

Numeriacl results for QPSK Random Interleaving

\begin{tabular}{|c|c|}
\hline $\mathrm{SNR}(\mathrm{dB})$ & QPSK (random interleaving) \\
\hline 0 & 0.5083 \\
\hline 2 & 0.4792 \\
\hline 4 & 0.4283 \\
\hline 6 & 0.3240 \\
\hline 8 & 0.1006 \\
\hline 10 & 0.0098 \\
\hline 12 & 0.0002 \\
\hline 14 & 0 \\
\hline 16 & 0 \\
\hline 18 & 0 \\
\hline
\end{tabular}

\section{OFDM with 16 QAM}

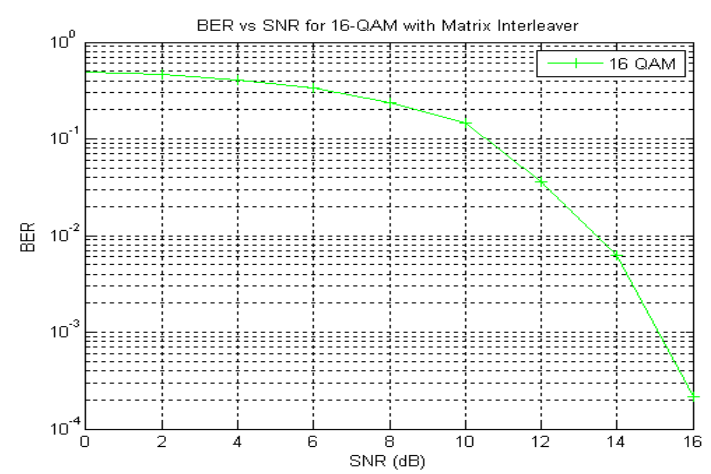

Fig. 12 BER vs. SNR for 16-QAM in OFDM with Matrix Interleaving

TABLE 8

Numerical results for 16-QAM in OFDM with Matrix Interleaving

\begin{tabular}{|c|c|}
\hline $\mathrm{SNR}(\mathrm{dB})$ & 16-QAM(Matrix Interleaver) \\
\hline 0 & 0.0049 \\
\hline 2 & 0.0046 \\
\hline 4 & 0.0041 \\
\hline 6 & 0.0033 \\
\hline 8 & 0.0027 \\
\hline 10 & 0.0015 \\
\hline 12 & 0.0003 \\
\hline 14 & 0 \\
\hline 16 & 0 \\
\hline 18 & 0 \\
\hline
\end{tabular}

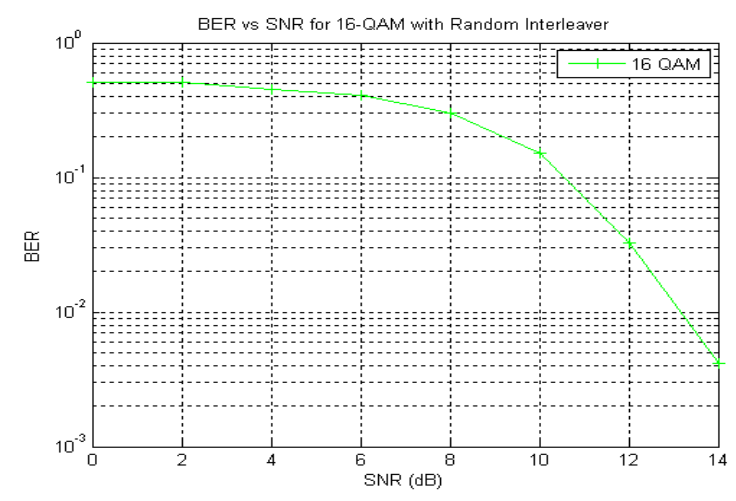

Fig. 12 BER vs. SNR for 16-QAM in OFDM with Random Interleaving TABLE 9

Numerical results for 16-QAM in OFDM with Random Interleaving

\begin{tabular}{|c|c|}
\hline SNR(dB) & 16-QAM (Random Interleaving) \\
\hline 0 & 0.0052 \\
\hline 2 & 0.0050 \\
\hline 4 & 0.0048 \\
\hline 6 & 0.0041 \\
\hline 8 & 0.0028 \\
\hline 10 & 0.0013 \\
\hline 12 & 0.0003 \\
\hline 14 & 0 \\
\hline 16 & 0 \\
\hline 18 & 0 \\
\hline
\end{tabular}

D. OFDM with 64-QAM

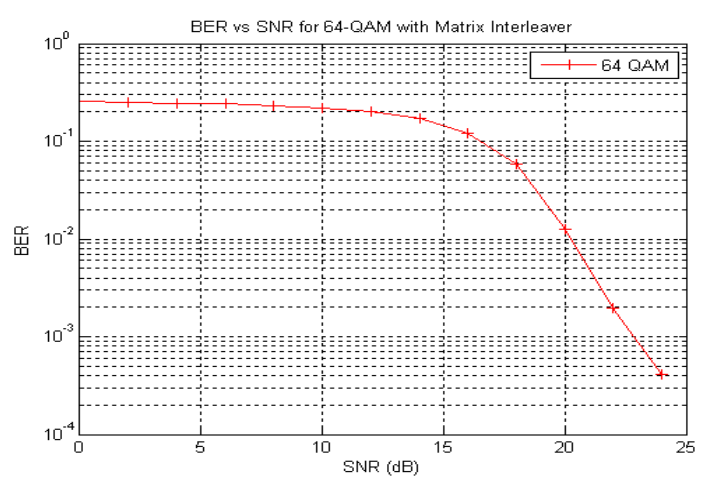

Fig. 13 BER vs. SNR for 64-QAM in OFDM with Matrix Interleaving

TABLE 10

Numerical results for 64-QAM in OFDM with Matrix Interleaving

\begin{tabular}{|c|c|}
\hline $\mathrm{SNR}(\mathrm{dB})$ & 64-QAM(Matrix Interleaver) \\
& \\
\hline 0 & 0.0025 \\
\hline 2 & 0.0025 \\
\hline 4 & 0.0025 \\
\hline 6 & 0.0024 \\
\hline 8 & 0.0023 \\
\hline 10 & 0.0022 \\
\hline 12 & 0.0020 \\
\hline
\end{tabular}




\begin{tabular}{|c|c|}
\hline 14 & 0.0017 \\
\hline 16 & 0.0011 \\
\hline 18 & 0.0006 \\
\hline 20 & 0.0002 \\
\hline 22 & 0 \\
\hline 24 & 0 \\
\hline
\end{tabular}

TABLE $12 \mathrm{~Eb} / \mathrm{N}_{0}$ required to achieve a PER $=10^{-2}$ (QPSK CONSTELLATION)

\begin{tabular}{|c|c|c|c|}
\hline \multirow{2}{*}{ Poruer } & \multicolumn{3}{|c|}{ Interlezver } \\
\cline { 2 - 4 } Allocation & RCPC-hesed & Adsptive Symhal [3] & Black Standard [5] \\
\hline Crpucity & 8.3 & 9.2 & 9.7 \\
Ro $_{0}$ & 7.8 & 8.5 & 8.9 \\
Unc. BER & 8.0 & 8.3 & 9.0 \\
MMSE & 8.2 & 8.5 & 9.0 \\
Liniform & 8.6 & 9.2 & 9.6 \\
\hline
\end{tabular}

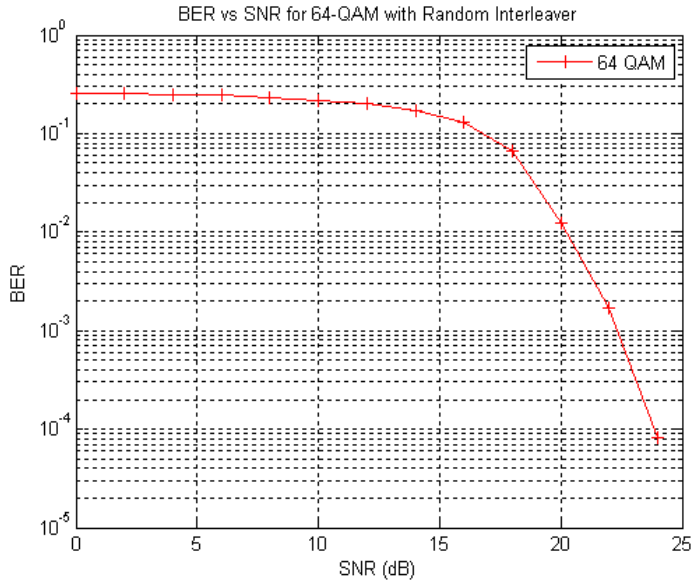

Fig. 14 BER vs. SNR for 64-QAM in OFDM with Random Interleaving TABLE 11

Numerical results for 64-QAM in OFDM with Random Interleaving

\begin{tabular}{|c|c|}
\hline \multirow{2}{*}{$\mathrm{SNR}(\mathrm{dB})$} & 64-QAM (Random Interleaving) \\
\hline 0 & 0.0026 \\
\hline 2 & 0.0025 \\
\hline 4 & 0.0025 \\
\hline 6 & 0.0024 \\
\hline 8 & 0.0023 \\
\hline 10 & 0.0022 \\
\hline 12 & 0.0020 \\
\hline 14 & 0.0018 \\
\hline 16 & 0.0013 \\
\hline 18 & 0.0007 \\
\hline 20 & 0.0001 \\
\hline 22 & 0 \\
\hline 24 & 0 \\
\hline &
\end{tabular}

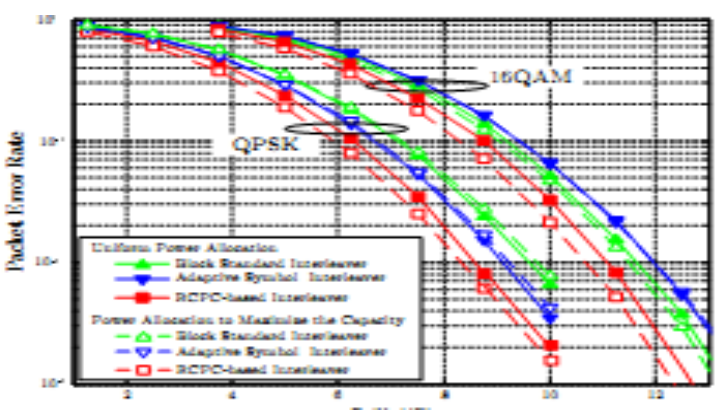

[1]

Fig. 15 PER comparision for different interleavers

\section{CONCLUSIONS}

In this paper simulation study of four modulation schemes (BPSK, QPSK, 16-QAM, 64-QAM) deployed over OFDM using convolutional code rate $1 / 2$ and $2 / 3$ with two different interleavers (Matrix and Random). Bit error rate (BER) is analyzed with respect to these modulation schemes over an AWGN channel. Motive of doing this simulation was to check the performance of these two interleavers in OFDM. As we know that the selection of efficient interleaver is a critical issue. From the above analysis of interleavers, the matrix interleaver outperforms in terms of BER, while random interleaver shows the low performance. So we can say that, matrix interleaver shows better performance with less BER. OFDM is computationally efficient due to the use of FFT techniques to implement modulation and demodulation functions.

We also observed from BPSK and QPSK BER plot that BER is less in case of BPSK for low SNR as compared to QPSK. Hence, high value of M-PSK increases spectrum efficiency, but easily affected by noise. So OFDM system with BPSK scheme is suitable for low capacity, short distance application. While the OFDM with higher M-ary modulation scheme is used for large capacity, long distance application at the cost of slight increase in SNR. The comparison of M-PSK and M-QAM indicates that, BER is large in M-PSK as compared to M-QAM and it generally depends on applications.

To compare our results we have also studied the results of different interleavers in OFDM with QPSK and 16 QAM from "Adaptive interleaver based on rate-compatible punctured convolutional codes" an IEEE transaction on communications, Vol.57, No. 6, June 2009.

At last we came to the point from our simulation and analytical study that the selection of interleaver is very important for communication system to get low BER. And as we know that low BER means that the system is working good as the erroneous bits are less. And for rate $1 / 2$ matrix interleaver is best to use as it is showing less BER at less SNR and at high SNR comparing to random interleaver. For code rate $2 / 3$ also matrix interleaver is best.

\section{ACKNOWLEDGMENT}

My deepest gratitude goes first and foremost to my project guide and supervisor Mr. Vivek Kumar Gupta, Assistant Professor, E.C.E. department, Dehradun Institute of Technology, Dehradun, for his constant support and guidance, and patience during the whole period of this paper.

Also, I would like to express my heartfelt gratitude to my dear friends whose continuous support, constructive suggestions and encouragement meant a lot in this work of mine.

My thanks would also go to my beloved family for their prayer, encouragement and support to achieve my goals. 


\section{References}

\section{Journal Papers:}

[1] F.Rey,M.Lamarca and G.Vazquez "Adaptive interleaver based on rate-compatible punctured convolutional codes",IEEE transactions on communications, vol.-57,no. 6,june-2009.

[2] K. Andrews, C. Heegard, and D. Kozen, "A theory of interleavers".Technical report TR97-1634,Department of Computer Science, Cornell University, June 1997.

[10] M.K.Gupta,V.Sharma "To improve BER of turbo coded OFDM channel over noisy channel" in journal of theoretical and applied information technology @ 2005-2009 JATIT.

[11] Md. Golam Rashed1"Transmission of Voice Signal: BER Performance Analysis of Different FEC Schemes Based OFDM System over Various Channels" International Journal of Advanced Science and Technology Vol. 34, September, 2011.

[4] L. Lou, Research Work on "Adaptive, turbo coded OFDM" (c) 2005.

\section{System Tools:}

[3] MATLAB, "Help, communication toolbox. Block interleaving"

\section{Books:}

[5] U. Dalal, "Wireless Communication" Chapter 9, wideband modulation techniques 2: OFDM (Multicarrier Modulation).page no. 382-384.

[6] B.P.Lathi, "Modern Digital and Analog Communication Systems" edition - 3,Chapter 16 ,"Error correcting codes",page no.747

[7] H.Taub,D.L.Schilling and G.Saha, "Taub's principles of communication systems" edition-3,Chapter 13, "Information theory and coding" page no. 682-684.

[10] T. S. Rappaport, Wireless Communications: Principles and Practice Englewood Cliffs, NJ: Prentice Hall, 1996.

[12] S. Hara and R. Prasad,"Multicarrier Techniques for $4 \mathrm{G}$ Mobile Communications", Artech House, edition-1, 2003.

[14] J. G. Proakis, Digital Communications, 4th ed. McGraw Hill,NY, 2001.

[9] Vocal TechnologiesLtd. Home page "802.11a White Paper".

\section{ABOUT AUTHOR}

Vineet Chaturvedi ${ }^{1}$, M.Tech. (Digital Communication), pursuing from Dehradun Institute of Technology, Dehradun.

Vivek Kumar Gupta ${ }^{2}$, Assistant Professor, Electronics and Communication Engineering Department, Dehradun Institute of Technology, Dehradun. 